Journal of Patient-Centered

Volume 2

Issue 4 - Integrative Medicine

Article 9

$11-20-2015$

\title{
Operating Room First Start Efficiency Throughout a Large Urban Hospital System
}

Callie Cox Bauer

Kiley A. Bernhard

Danielle M. Greer

Scott Kamelle

Follow this and additional works at: https://aah.org/jpcrr

Part of the Health and Medical Administration Commons, and the Surgery Commons

\section{Recommended Citation}

Cox Bauer C, Bernhard KA, Greer DM, Kamelle S. Operating room first start efficiency throughout a large urban hospital system. J Patient Cent Res Rev. 2015;2:202-203. doi: 10.17294/2330-0698.1219

Published quarterly by Midwest-based health system Advocate Aurora Health and indexed in PubMed Central, the Journal of Patient-Centered Research and Reviews (JPCRR) is an open access, peer-reviewed medical journal focused on disseminating scholarly works devoted to improving patient-centered care practices, health outcomes, and the patient experience. 


\section{Proceedings of 2015 Aurora Scientific Day}

The following abstracts were presented at the 41st annual Aurora Scientific Day research symposium, held May 20, 2015, at Aurora Health Care Conference Center in Milwaukee, Wisconsin. Aurora Scientific Day provides a forum for original research conducted by residents, fellows, students, teaching and research faculty, and other allied health professionals at Aurora Health Care, a private nonprofit health care provider with 15 hospitals, 150 clinics and 70 pharmacies integrated throughout eastern Wisconsin and northern Illinois.

\section{FIRST PLACE ORAL PRESENTATION Model Assessment and Development of Risk Stratification of Surgical Site Infection Following Cesarean Delivery for a High-Risk, Urban Population}

Dakisha N. Lewis, Nicole P. Salvo, Kiley A. Bernhard, Danielle M. Greer

Department of Obstetrics and Gynecology, Aurora Sinai Medical Center and Aurora UW Medical Group; Center for Urban Population Health

Background: Surgical site infection (SSI) remains a major cause of morbidity despite efforts aimed at prevention and treatment. Risk stratification tools identify patients at greatest risk of SSI. Two models of stratification are: 1) the Centers for Disease Control and Prevention's National Healthcare Safety Network SSI Risk Index (NHSN), which assigns risk based on surgery duration, surgical wound contamination and physical status; and 2) the New Risk Stratification Schema (NRSS). The NRSS aimed to improve upon NHSN by incorporating five variables: diabetes control, body mass index (BMI), chorioamnionitis, methods of placental extraction and skin closure.

Purpose: Our objectives were to: 1) compare and evaluate NHSN and NRSS in terms of risk stratification outcomes in a high-risk urban population; and 2) develop a risk stratification model appropriate for assessing SSI risk in our population.

Methods: Data-related risk factors were gathered through chart review of all women who underwent cesarean delivery from September 2012 to October 2013. Using NHSN and NRSS models, we classified patients by risk of SSI following cesarean delivery. Logistic regression model effects represented 12 a priori risk factors in SSI, including BMI, diabetes, chorioamnionitis, delivery indication, use of chlorhexidine, preoperative antibiotics, timing of antibiotics, manual placental removal, antibiotic re-dosing, incision closure via staples, number of people present and surgery duration. Model-derived predicted values of SSI were used to stratify patients into low-, moderate- and high-risk categories. Strength of associations between SSI outcome and classified risks were examined. Agreement in risk classification between NHSN and NRSS, and each with our model, were assessed.

Results: Patients were normally distributed across the low- $(21.3 \%)$, moderate- $(55.9 \%)$ and high-risk $(22.9 \%)$ categories of NHSN, but under NRSS were restricted to moderate- $(33.6 \%)$ and high-risk (66.4\%) categories. While both methodologies produced results strongly associated with SSI $(\mathrm{P}<0.0001)$, agreement in SSI risk occurred for only $46.3 \%$ of patients. Modeling efforts established chorioamnionitis, BMI and surgery duration as the three most significant predictors of SSI.

Conclusion: While both NHSN and NRSS produced results strongly associated with SSI, distribution of patients was shifted toward high-risk in the NRSS arm. Our stratification model is a simplification of the NRSS, utilizing only three highly significant predictors: chorioamnionitis, BMI and surgery duration.

\section{SECOND PLACE ORAL PRESENTATION Operating Room First Start Efficiency Throughout a Large Urban Hospital System}

Callie Cox Bauer, Kiley A. Bernhard, Danielle M. Greer, Scott Kamelle

Department of Obstetrics and Gynecology, Aurora Sinai Medical Center; Center for Urban Population Health; Gynecologic Oncology, Aurora Health Care

Background: Operating room delays decrease health care system efficiency and increase hospital costs. Data on delays in a multihospital system are sparse.

Purpose: In an effort to improve our operating room efficiency, we investigated operating room delays, the causes and the impending financial impact.

Methods: A retrospective analysis on first case-of-the-day surgeries at three hospitals during 2013 was conducted. Delays were defined as in-room time being after scheduled surgery start time. Length of delay and causes were recorded. Patient demographics, body mass index, hospital facility, total number of procedures, provider specialty and time of patient arrival were incorporated into a logistic regression model to identify significant variables. Hosmer-Lemeshow was used to measure goodness-of-fit and predictive power. Cost was calculated using published estimates.

Results: 5,607 cases were examined and $88 \%$ were delayed. Surgeons (21\%), anesthesiologists $(6.17 \%)$, patients $(5.42 \%)$, staff $(3.60 \%)$, facility $(2.10 \%)$ and other $(2.35 \%)$ were identified as causes. Mean time for patient arrival to surgery was $104.57 \mathrm{~min}$. Mean time between arrival and 
room placement was $127.38 \mathrm{~min}$. The average delay time from scheduled surgery start was $24.26 \mathrm{~min}$. Logistic regression identified hospital facility $(\mathrm{P}<0.0001)$, surgical specialty $(\mathrm{P}<0.0001)$, patient age $(\mathrm{P}=0.0004)$ and late patient arrival $(\mathrm{P}=0.0005)$ as significant predictors of delay. Operating room delays were responsible for $\$ 444,074$ in lost revenue.

Conclusion: In our study, $88 \%$ of first start cases were delayed, the majority of which were caused by the surgeon. However, hospital facility, surgical subspecialty, patient age and arrival time also significantly affected delays. Correction of operating room delays can significantly reduce hospital costs.

\section{THIRD PLACE ORAL PRESENTATION}

Assessing the Effectiveness of Implementation of Unified Workflow in Improvement of Medication Reconciliation for Aurora St. Luke's Family Medicine Residency Outpatients

Katherine Meyers, Jessica Konarske, Jessica J.F. Kram, Dennis J. Baumgardner

Department of Family Medicine, Aurora UW Medical Group, Aurora Health Care; Center for Urban Population Health

Background: Medication errors are the most common errors occurring in hospitals. Preventable adverse drug events are linked with 1 in 5 injuries or deaths; $23 \%$ of medication errors in primary care occur due to inaccuracies in the medication list. Quality improvement projects designed to improve accuracy of outpatient medication reconciliations may decrease the number of medication errors and increase patient safety by preventing adverse drug events.

Purpose: To determine whether a unified workflow for medication reconciliation improves the accuracy of ambulatory, electronic medical record (EMR)-based patient medication records.

Methods: Retrospective study of random sample of patients from Aurora Family Medicine Residency Clinics before (prior to March 31, 2014) and after (December 10, 2014) improvements to the medication reconciliation process $(n=80$ and $n=77$, respectively). Aurora pharmacy medication lists were obtained and compared to that of the EMR. To preserve patient and caregiver confidentiality, charts were assigned arbitrary identifiers. Two-sample t-tests were used to compare pre- and post-medication reconciliation. An additional patient chart audit on pre- $(n=51)$ and post- $(n=45)$ workflow implementation to assess utilization of workflow was conducted; Fisher's exact tests were used to gauge changes $(\mathrm{P}<0.05)$.

Results: When comparing pre- and post-medication reconciliation implementation, there was a significant decrease in the number of EMR medications not on the pharmacy list (mean 0.475 vs. $0.208 ; \mathrm{P}=0.022$ ). Number of providers reviewing the EMR medication record improved significantly by $30.4 \%(\mathrm{P}=0.045)$. A downward trend in the number of unintentional medication duplicates also was observed by a $13.3 \%$ decrease $(\mathrm{P}=0.07)$.

Conclusion: Implementation of systematic workflow and care team education led to overall improvement in accuracy of EMR medication reconciliation. This quality improvement project led to identification of multiple barriers to accuracy. Future areas of focus would include continued education around current workflow and additional attention to medication compliance via out-of-date prescriptions.

\section{FIRST PLACE POSTER (tie)}

Using an Automated Model to Identify Older Patients at Risk for 30-Day Hospital Readmission and 30-Day Mortality

Ariba Khan, Mary L. Hook, Maharaj Singh, Marsha Vollbrecht, Aaron Malsch, Michael L. Malone

Department of Geriatrics, Aurora UW Medical Group; Knowledge-Based Nursing Department, Aurora Health Care; Aurora Research Institute, Aurora Health Care; Senior Services, Aurora Health Care

Background: A real-time electronic health record (EHR) predictive model that identifies older patients at risk for readmission and mortality may assist the health care team in improved patient care.

Purpose: This study was performed to generate an automated 30-day readmission and 30-day mortality risk model using data from the EHR in hospitalized older adults.

Methods: This was a retrospective cohort study. Included were patients age 65 years and older admitted to the hospital from July 2012 to December 2013. An automated predictive model was derived from variables collected from the EHR including socioeconomic factors, medical diagnoses and health care utilization. The study sample was randomly divided into derivation $(70 \%)$ and validation $(30 \%)$ cohorts. Multiple logistic regression analysis was performed to derive a prediction model. A scoring system was developed for estimating risk of 30-day readmission.

Results: The study included 11,223 patients in one hospital, of which $46 \%$ were male, $20 \%$ were age $>85$ years, $6.2 \%$ were black, $60 \%$ required emergency admission, $2.8 \%$ required an ICU stay and $62.7 \%$ were discharged home. Overall 30 -day readmission and mortality rates were $13.7 \%$ and $1.5 \%$. The risk model predicted 30-day readmission, with c-statistics of 0.62 (95\% confidence interval [CI]: $0.61-0.64)$ and 0.62 (95\% CI: $0.60-0.65)$ in the derivation and validation cohorts, respectively. A readmit risk score was developed that ranged from 0 to 20 . The readmission rate increased as the score increased: score $0-4$, readmission rate $=8.38 \%$; score $5-9$, readmission rate $=13 \%$; and score $>10$, readmission rate $20 \%$ $(\mathrm{P}<0.0001)$ in the derivation cohort. Results were similar for validation cohort. The risk model predicted 30-day all-cause mortality with c-statistics of 0.81 (95\% CI: $0.77-0.86)$ and 0.73 (95\% CI: $0.66-0.81)$ in the derivation and validation cohorts. The variables associated with mortality included discharge to nursing home, urgent admission status, social 